\title{
NONSTANDARD TOPOLOGY ON FUNCTION SPACES WITH APPLICATIONS TO HYPERSPACES
}

\author{
HERMANN RENDER
}

\begin{abstract}
In this paper the techniques of Nonstandard Analysis are used to study topologies on the set of all continuous functions. We obtain nonstandard characterizations for conjoining and splitting topologies and we give a complete description of the monads of the compact-open topology which leads to very elegant and simple proofs of some important results. For example we prove a generalized Ascoli Theorem where the image space is only Hausdorff or regular. Then we apply our results to the hyperspace and solve questions of Arens and Dugundji, Wattenberg and Topsøe. Finally we discuss real compact spaces and the continuity of the diagonal function.
\end{abstract}

\section{INTRODUCTION}

Let $X, Y$ be topological spaces. The most important topology on the set $C(X, Y)$ of all continuous functions is surely the compact-open topology denoted by $\tau_{k}$. We give a complete description of the monads of $\tau_{k}$ if $X$ or $Y$ possesses a weak separation property. Surprisingly this result is not true for general spaces contradicting the main result in [6]. Recall that $X$ is a $k$-space if the topology $k(X):=\{U \subset X: U \cap K$ is relatively open in $K$ for every compact set $K\}$ coincides with the original topology. It is well known that every topological space with a countable neighborhood base at each point (also called an $A 1$-space), in particular every metric space is a $k$-space. For the large class of all $k$-spaces we obtain a nice characterization of the nearstandard points of $\tau_{k}$. This enables us to give a very elegant nonstandard proof of the Ascoli Theorem for $k$-spaces (or more general, for $k_{3}$-spaces) and we show that the regularity of $Y$ can be replaced by a weaker separation property which we have called prehausdorffness. Moreover it follows from our proofs that a result in [18] is not correct.

In homotopy theory the following problem is important: Let $f: T \times X \rightarrow Y$ be a continuous function where $T$ is an arbitrary topological space. Characterize the topologies $\tau$ on $C(X, Y)$ such that every "path" $f^{\mu}$ is a continuous function from $T$ into $C(X, Y)$ where $f^{\mu}(t)(x):=f(t, x)$. A topology $\tau$

Received by the editors November 13, 1990.

1991 Mathematics Subject Classification. Primary 54J05; Secondary 54C35.

Key words and phrases. Hyperspaces, convergence topology, Ascoli Theorem, diagonal function, exponential map, real compactness. 
satisfying this condition is called splitting. Conversely, $\tau$ is called conjoining if for every topological space $T$ the continuity of $f^{\mu}: T \rightarrow C(X, Y)$ implies the continuity of $f$. We give nonstandard characterizations for a conjoining or splitting topology. As a consequence we obtain that a topology is splitting if and only if it is coarser than every conjoining topology. This fact is very useful for applications to the set $\mathscr{P}_{\mathrm{Pl}}(X)$ of all closed subsets, the so-called hyperspace: it is well known that $\mathscr{P}_{\mathrm{cl}}(X)$ can be identified with $C(X,\{0,1\})$ if $Y:=\{0,1\}$ is endowed with the Sierpinski topology $\{\varnothing,\{0\}, Y\}$. Arens and Dugundji have asked whether the Vietoris topology is the smallest conjoining Hausdorff topology for a compact Hausdorff space $X$. By our results this is true if and only if $X$ is discrete. Furthermore we show that the convergence topology coincides with the so-called $N$-compact topology introduced by L. Narens. In general, the union of two sets is not a continuous operation for the convergence topology answering a question of Wattenberg negatively. For the class of all topological vector spaces we can show that the convergence topology coincides with the Fell topology if and only if the space is locally compact. This partially answers a question of Topsøe in [3]. For the class of all metric spaces we prove that $\mathscr{P}_{\mathrm{cl}}(X)$ is an $A 1$-space for the convergence topology if and only if $X$ is a locally compact space with a countable base. Then we give a nonstandard characterization of real compact spaces using the methods developed in [5] and give some nonstandard proofs of known results. In the last section we extend some results of $\mathrm{R}$. McCoy concerning the continuity of the diagonal function.

The paper is divided into seven sections. In the first section we discuss topological spaces which satisfy a weak separation property (cf. formula (1)). In [23] these spaces are called $R_{1}$-spaces but we prefer the more suggestive notion of a prehausdorff space. The reader who is not interested in such a specialized separation property may skip the first section and need only know that every regular space and every Hausdorff space is prehausdorff. The second section deals with the compact-open topology and contains some further results. The third section is devoted to the Ascoli Theorem; an example shows that our results are the best possible. The next section contains the characterizations of a conjoining or splitting topology and an elegant proof of the so-called exponential law. In the fifth section, perhaps the main part, we present the results about hyperspaces. Then we prove the nonstandard characterization of real compactness. In the last section we study the continuity of the diagonal function and some related questions.

Now let us introduce some terminology. We always assume a polysaturated nonstandard model. If $(X, \tau)$ is a topological space then $m(x):=$ $\bigcap_{U \in \tau, x \in U}{ }^{*} U$ is the monad of $x \in X$. Frequently we write $y \approx x$ instead of $y \in m(x)$. As usual $\mathrm{ns}^{*} X:=\bigcup_{x \in X} m(x)$ is the set of all nearstandard points and $\mathrm{cpt}^{*} X:=\bigcup_{K \subset X \text { compact }}^{*} K$ the set of all compact points. If $z \in{ }^{*} X$ then $\mu(z):=\bigcap_{U \in \tau,}, \in^{*}{ }^{*} U$ is the monad of $z \in{ }^{*} X$. For $A \subset X$ let ${ }^{\sigma} A:=\left\{{ }^{*} a: a \in A\right\}$. Moreover we assume elementary knowledge of nonstandard topology as presented in [12]. For topological definitions we refer to [13]. Recall that a $T_{1}$-space is a topological space such that every point is closed. A topological space is regular if every closed set and every point outside from it can be separated by disjoint open sets. If $X, Y$ are sets $F(X, Y)$ denotes the set of all functions $f: X \rightarrow Y$. 


\section{Prehausdorff SPaCes}

Prehausdorff spaces generalize both Hausdorff spaces and regular spaces and a simple nonstandard definition is given by formula (1). Roughly speaking a topological space $X$ is prehausdorff if distinct points can either be identified or separated. More precisely: define a reflexive and transitive relation $\sim$ on $X \times X$ where $y \sim x$ means that $x \in U$ implies $y \in U$ for every open set $U$; cf. [9]. Then $X$ is prehausdorff if $\sim$ is an equivalence relation and the quotient space $X / \sim$ is Hausdorff. Note that $y \sim x$ iff ${ }^{*} y \approx x$ for $x, y \in X$. As indicated in [32, p. 199] a space $X$ is prehausdorff if and only if for all $x, y \in X$ the following condition holds:

$$
m(x) \cap m(y) \neq \varnothing \Rightarrow^{*} x \approx y .
$$

Using (1) we can extend the relation $\approx \subset \mathrm{ns}^{*} X \times^{\sigma} X$ to an equivalence relation $\approx$ on $\mathrm{ns}^{*} X \times \mathrm{ns}^{*} X$ : for $x, y \in \mathrm{ns}^{*} X$ define $y \approx x$ to mean that there exists $c \in X$ with $y \approx c$ and $x \approx c$. Hence $X$ is prehausdorff iff $\approx$ can be extended to an (unique) equivalence relation on $\mathrm{ns}^{*} X \times \mathrm{ns}^{*} X$. It is clear that $\approx$ is extendible to a nuclear (cf. $\left[17\right.$, p. 18]) equivalence relation on ${ }^{*} X \times^{*} X$ iff $X$ is uniformizable. Therefore it is a natural question whether $\approx$ can be extended at all to a nuclear relation on ${ }^{*} X \times{ }^{*} X$ : consider the filter $\mathscr{U}_{\Delta}$ of all neighborhoods of the diagonal in $X \times X$ and define $y \approx_{\Delta} x$ to mean that $(x, y) \in{ }^{*} U$ for all $U \in \mathscr{U}_{\Delta}$. Obviously $\approx_{\Delta}$ is reflexive and symmetric. It is easy to see that $\approx_{\Delta}$ is an extension of $\approx$ if $X$ is prehausdorff. Consequently, if $X$ is compact and prehausdorff then $\approx_{\Delta}$ is an equivalence relation on $\mathrm{ns}^{*} X={ }^{*} X$. Thus we have shown

1.1. Theorem. Let $X$ be compact. Then $X$ is prehausdorff if and only if it is uniformizable.

\subsection{Corollary. A compact subspace of a prehausdorff space is regular.}

Thus a locally compact space is prehausdorff iff it is regular. It is obvious that a $T_{1}$-space is prehausdorff iff it is Hausdorff. Using (1) it is easy to see that prehausdorffness is a productive property. Consequently there exist prehausdorff spaces being neither regular nor Hausdorff.

1.3. Proposition. Let $X$ be prehausdorff and $K \subset X$ compact. Then the closure $\bar{K}$ is compact.

Proof. Theorem 8.3.8 in [32] yields $\bar{K}=$ st ${ }^{*} K:=\left\{x \in X: \exists y \in{ }^{*} K\right.$ with $y \approx x\}$. Let $\left(U_{i}\right)_{i \in I}$ be an open covering of st $^{*} K$. It is easy to see that ${ }^{*} K \subset \bigcup_{i \in I}{ }^{*} U_{i}$. By saturation we obtain ${ }^{*} K \subset \bigcup_{k=1}^{n}{ }^{*} U_{i_{k}}$. Now it suffices to show that st $^{*} K \subset \bigcup_{k=1}^{n} U_{i_{k}}$; for $x \in \mathrm{st}^{*} K$ there exists $z \in{ }^{*} K$ with $z \approx x$. Since $K$ is compact there exists $y \in K$ with $z \approx y$. By (1) we have ${ }^{*} x \approx y \in$ $K \subset \bigcup_{k=1}^{n} U_{i_{k}}$. Thus $x \in U_{i_{k}}$ for some $k \in\{1, \ldots, n\}$ since all $U_{i_{k}}$ are open. The proof is complete.

It is well known that the compactness of $K$ in Proposition 1.3 cannot be weakened to the condition ${ }^{*} K \subset \mathrm{ns}^{*} X$, even for Hausdorff spaces; cf. [17, p. 31]. We note in passing that the condition ${ }^{*} A \subset \mathrm{ns}^{*} X$ for a subset $A \subset X$ is equivalent to the boundedness of $A$, i. e., that every open covering of the whole space $X$ contains a finite covering of the set $A$; cf. [15] for a standard treatment. For a survey about other separation properties we refer to [11]. 


\section{COMPACT-OPEN TOPOLOGY}

Let $X, Y$ be topological spaces and let $H$ be a subspace of $C(X, Y)$. If $\alpha$ is a system of subsets of $X$ then the set-open topology $\tau_{\alpha}$ on $H$ is generated by the sets $[A, V]:=\{f \in H: f(A) \subset V\}$ with $A \in \alpha$ and $V \subset Y$ open. Obviously one can assume that $\alpha$ is closed under finite unions. If $\alpha$ is the family of all closed subsets then $\tau_{\alpha}$ is called the closed-open topology, if $\alpha$ is the system $k$ of all compact subsets then $\tau_{k}$ is called the compact-open topology. At first we give a characterization of the monad relation $\approx_{k}$ of the compactopen topology: if $X$ or $Y$ is prehausdorff then the following statement is valid for $f \in{ }^{*} C(X, Y)$ and $f_{0} \in C(X, Y)$ :

$$
f \approx_{k} f_{0} \Leftrightarrow \text { For all } x \in \operatorname{cpt}^{*} X, x_{0} \in X, x \approx x_{0} \text { is } f(x) \approx f_{0}\left(x_{0}\right) .
$$

The proof of (2) follows by a modification of the proof of Proposition 1.17 in [14]. Essentially one uses the fact that every compact set is a regular subspace; such spaces are called $K R$-spaces in [16]. Another sufficient condition for the validity of (2) is basic local compactness of $X$, i. e., that every point $x \in X$ has a neighborhood system consisting of compact sets. Recall that local compactness only means that every point has a compact neighborhood.

Using the nonstandard characterization of continuity it is obvious that (2) is equivalent to the continuity of the evaluation $e: C(X, Y) \times K \rightarrow Y$ defined by $e(f, x):=f(x)$ for every compact set $K \subset X$. Thus we obtain from (2) the following result:

2.1. Theorem. Let $X$ be basic locally compact or let $X$ be locally compact and $Y$ prehausdorff. Then the evaluation $e: C(X, Y) \times X \rightarrow Y$ is continuous for the compact-open topology.

We emphasize that (2) is not valid for arbitrary spaces even if $X$ and $Y$ are compact; cf. Theorem 5.7 and Example 99 in [31]. Hence Theorem A in [6] is false.

Recall that a $k_{Y}$-space is a topological space $X$ on which a $Y$-valued function is continuous if its restriction to each compact subspace is continuous; see, e.g., [20, p. 74]. A space is a $k$-space $\left(k_{3}\right.$-space) if it is a $k_{Y}$-space for every topological (regular) space $Y$. Considering the identity map id $:(X, \tau) \rightarrow$ $(X, k(X))$ one obtains the definition of a $k$-space given in the introduction.

One of the most striking tools in nonstandard analysis is taking standard parts of objects of the nonstandard universe. If $Y$ is Hausdorff we can define for every $f \in{ }^{*} C(X, Y)$ with $f\left({ }^{*} x\right) \in \mathrm{ns}^{*} Y$ for all $x \in X$ the standard part function st $f: X \rightarrow Y$ by st $f(x):=$ st $_{Y}\left(f\left({ }^{*} x\right)\right)$ where st ${ }_{Y}$ denotes the standard part map of $Y$. Thus we have a function

$$
\text { st : }\left\{f \in{ }^{*} C(X, Y): f\left({ }^{*} x\right) \in \mathrm{ns}^{*} Y \text { for all } x \in X\right\} \rightarrow F(X, Y) \text {. }
$$

It is well known that st $f$ is in general not a continuous function. Indeed, let $X$ be a completely regular Hausdorff space and $Y=\mathbf{R}$; we show that st is surjective: let $f: X \rightarrow \mathbf{R}$ be a function. For every finite set $E \subset X$ the restriction $f: E \rightarrow \mathbf{R}$ is continuous. Since compact sets are $C$-embedded we can extend $f_{\mid E}$ to a continuous function $g: X \rightarrow \mathbf{R}$; cf. [10]. Now apply a saturation argument to the system of all sets $S_{E}:=\left\{g \in{ }^{*} C(X, \mathbf{R}): g\left({ }^{*} x\right)=\right.$ ${ }^{*} f\left({ }^{*} x\right)$ for all $\left.x \in E\right\}$ with $E \subset X$ finite. 
Even in the non-Hausdorff case we can define standard parts: let $f:{ }^{*} X \rightarrow{ }^{*} Y$ be a function with $f\left({ }^{*} x\right) \in \mathrm{ns}^{*} Y$. Define $g: X \rightarrow Y$ by $g(x):=y$ for some $y \in Y$ with $f\left({ }^{*} x\right) \approx y$. Every such $g$ is called a standard part function of $f$. We call an internal function $f:{ }^{*} X \rightarrow{ }^{*} Y$ ns-continuous (cpt-continuous resp.) if (i) $f\left({ }^{*} x_{0}\right) \in \mathrm{ns}^{*} Y$ for all $x_{0} \in X$ and (ii) for every $x_{0} \in X, y \in Y$, $f\left({ }^{*} x_{0}\right) \approx y$ implies $f(x) \approx y$ for all $x \in \mathrm{ns}^{*} X$ (cpt ${ }^{*} X$ resp.) with $x \approx x_{0}$. If $Y$ is prehausdorff the second rather technical condition can be expressed to: $x \approx x_{0}$ implies $f(x) \approx f\left({ }^{*} x_{0}\right)$ for every $f \in{ }^{*} H$ where $\approx$ is the extended equivalence relation.

The following proposition is well known and its proof is very similar to that of Proposition 2.2.

2.1. Proposition. Let $Y$ be regular and $f:{ }^{*} X \rightarrow{ }^{*} Y$ be ns-continuous. Then every standard part function of $f$ is continuous.

2.2. Proposition. Let $X$ be a $k_{Y}$-space and $f:{ }^{*} X \rightarrow{ }^{*} Y$ be cpt-continuous. Then every standard part function of $f$ is continuous provided that one of the following conditions is satisfied:

(a) $Y$ is prehausdorff and $f\left({ }^{*} K\right) \subset \mathrm{cpt}^{*} Y$ for every compact set $K \subset X$.

(b) $Y$ is regular.

Proof. It suffices to show that every standard part function $g: X \rightarrow Y$ of $f$ is continuous on every compact set $K \subset X$. Since $f\left({ }^{*} K\right) \subset \mathrm{cpt}^{*} Y$ a straightforward saturation argument shows that there exists a compact set $L \subset Y$ with $f\left({ }^{*} K\right) \subset{ }^{*} L$. By Proposition 1.3 we can assume that $L$ is closed. Let $x_{0} \in K$ and $V$ be a neighborhood of $g\left(x_{0}\right)$. Choose an open neighborhood $V_{2}$ of $g\left(x_{0}\right)$ such that $g\left(x_{0}\right) \in V_{2} \cap L \subset \bar{V}_{2} \cap L \subset V$; cf. Corollary 1.2. Since $f$ is cpt-continuous there exists a neighborhood $U$ of $x_{0}$ with $f\left({ }^{*} U \cap{ }^{*} K\right) \subset{ }^{*} V_{2}$. It suffices to show that $g(U \cap K) \subset V$. Let $x \in U \cap K$. Then $f\left({ }^{*} x\right) \in{ }^{*} V_{2}$. Since $f\left({ }^{*} x\right) \approx g(x)$ we have $g(x) \in \bar{V}_{2}$ and similarly $g(x) \in \bar{L}=L$. Thus $g(x) \in V$. For (b) repeat the proof with $L=Y$.

Now let $Y$ be a regular space. Using Proposition 2.1 and formula (2) it is now easy to see that

$$
\begin{aligned}
\{f: f \text { ns -continous }\} & \subset \mathrm{ns}_{\tau_{k}}{ }^{*} C(X, Y) \\
& \subset\left\{f \in{ }^{*} C(X, Y): f \text { cpt-continuous }\right\} .
\end{aligned}
$$

The latter inclusion is an equality if $X$ is a $k_{Y}$-space as Proposition 2.2 shows. Thus we have a description of the nearstandard points of $C(X, Y)$ for a large class of topological spaces. Of course, for a locally compact space we obtain the well-known characterization that $f \in \mathrm{ns}_{\tau_{k}}{ }^{*} C(X, Y)$ iff it is ns-continous; cf. Proposition 2.13 in [1], i.e., that the first inclusion in (4) is an equality.

We want to prove partial converses of the two last statements. If $C(X, Y)$ consists only of constant functions then we have in (4) equality independent of the topological structure of $X$. Thus we need some assumptions to guarantee the existence of enough continuous functions. The most common is to require that $(X, \tau)$ is a completely regular Hausdorff space and $Y$ a topological $T_{1}$ space containing a nontrivial path. Then it is easy to see that $H:=C(X, Y)$ is a completely regular family in the sense of the following

Definition. Let $X, Y$ be topological spaces. Then $H \subset F(X, Y)$ is a completely regular family if there exists $y_{0} \neq y_{1} \in Y$ such that (i) for every closed 
set $A \subset X$ and every $z \in X \backslash A$ there exists $f \in H$ with $f(A)=\left\{y_{0}\right\}$ and $f(z)=y_{1}$, (ii) the constant function $\hat{y}_{0}: X \rightarrow Y$ defined by $\hat{y}_{0}(x)=y_{0}$ is in $H$ and (iii) ${ }^{*} y_{1} \notin m\left(y_{0}\right)$.

For example, $C(X, \mathbf{R})$ is a completely regular family iff $X$ is completely regular. If $Y:=\{0,1\}$ is endowed with the Sierpinski topology $\{\varnothing,\{0\}, Y\}$ then $C(X,\{0,1\})$ is a completely regular family provided that $X$ is regular or a $T_{1}$-space. Another class of examples occur in Banach algebra theory: a complex commutative Banach algebra $A$ is called completely regular (cf. [29, p. 174]) if the image of $A$ under the Gelfand transformation is a completely regular family in our sense.

2.3. Proposition. Let $H \subset F(X, Y)$ be a completely regular family. For any $z \in{ }^{*} X$ there exists a peak function for the point $z \in{ }^{*} X$, i. e., a function $f \in{ }^{*} H$ with $f(z)={ }^{*} y_{1}$ and $f(x)={ }^{*} y_{0}$ for all $x \in{ }^{*} X \backslash \mu(z)$.

Proof. Transfer of the condition (i) shows that $S_{V}:=\left\{f \in{ }^{*} H: f\left({ }^{*} X \backslash^{*} V\right)=\right.$ $\left\{{ }^{*} y_{0}\right\}$ and $\left.f(z)={ }^{*} y_{1}\right\}$ is nonempty for every $V \in \tau$ with $z \in V$. Now use saturation.

Let $C(X, Y)$ be a completely regular family and $X$ prehausdorff. We show that the first inclusion in (4) is an equality iff $X$ is locally compact: If $X$ is not locally compact there exists $z \in \mathrm{ns}^{*} X \backslash \mathrm{cpt}^{*} X$. Then the peak function $f$ for the point $z$ is not ns-continuous but $f \approx_{k} \hat{y}_{0}$, use formula (2) and $\mu(z) \subset^{*} X \backslash \mathrm{cpt}^{*} X$.

Now let $X$ be a completely regular Hausdorff space and $Y=\mathbf{R}$. We show that the second inclusion in (4) is an equality iff $X$ is a $k_{\mathbf{R}}$-space: let $f: X \rightarrow \mathbf{R}$ be continuous on each compact set $K \subset X$. As above it follows that $S_{K}:=$ $\left\{g \in{ }^{*} C(X, \mathbf{R}): g(x)={ }^{*} f(x)\right.$ for all $\left.x \in{ }^{*} K\right\}$ is nonempty. By saturation there exists $g \in{ }^{*} C(X, \mathbf{R})$ with $g(x)={ }^{*} f(x)$ for all $x \in \mathrm{cpt}^{*} X$. Since $g$ is cpt-continuous there exists by assumption a continuous standard function which coincides with $f$. Thus $f$ is continuous.

Our next example shows that the assumptions in Propositions 2.1 and 2.2 cannot be omitted. Moreover, it is interesting for the next section.

2.4. Example. Let $\tau$ be the usual topology on the complex plane $\mathbf{C}$ and let $\tilde{\tau}$ be the (finer) topology generated by the sets $U_{n}:=U \backslash\{1 / k: k \geq n\}$ and $U$ with $U \in \tau, n \in \mathbf{N}$. Define $f_{t}: \mathbf{R} \rightarrow(\mathbf{C}, \tilde{\tau})$ by $f_{t}(x):=e^{i t} x$ with $t \in(0,1]$. It is straightforward to verify that every ${ }^{*} f_{t}$ with $t \in{ }^{*}(0,1]$ is ns-continuous. But for $t \approx_{\mathbf{R}} 0$ the standard part function $g(x)=x$ is not continuous.

The concept of equicontinuity of a family $H \subset C(X, Y)$ requires a uniform structure for $Y$. The weaker concept of even continuity is a purely topological notion but rather technical: recall that $H$ is evenly continuous if for every $x_{0} \epsilon$ $X, y \in Y$, and for every neighborhood $V$ of $y$ there exists a neighborhood $U$ of $x$ and a neighborhood $W$ of $y$ such that $f\left(x_{0}\right) \in W$ implies $f(U) \subset V$ for all $f \in H$. Proposition III 8.7 in [12] shows that a family $H \subset C(X, Y)$ is evenly continuous (evenly continuous on compacta) iff every $f \in{ }^{*} H$ satisfies condition (ii) of ns-continuity (cpt-continuity). If we assume that $Y$ is a compact Hausdorff space this means that

$$
\bigcup_{H \subset C(X, Y) \text { evenly continuous }}{ }^{*} H \subset\left\{f \in{ }^{*} C(X, Y): f \text { is ns-continuous }\right\} .
$$


It is natural to ask when this inclusion is an equality. We assume that $X$ is a completely regular Hausdorff space and $Y$ contains a nontrivial path $\gamma$ with $\gamma(0)=: y_{0}$. Let $\tau_{y_{0}}$ be the system of all open neighborhoods of $y_{0}$. One can prove that for every $V \in \tau_{y_{0}}$ the set $[X, V]$ is a completely regular family. We show that in (5) equality holds iff $X$ is discrete. The sufficiency is obvious. Observe that the set on the right side in (5) contains $m\left(\widehat{y}_{0}\right):=\bigcap_{V \in \tau_{y_{0}}}{ }^{*}[X, V]$. If we have equality in (5) then the saturation principle shows that $[X, V] \subset H$ for an evenly continuous family $H$, in particular $[X, V]$ is evenly continuous. But a completely regular family $H \subset C(X, Y)$ is evenly continuous iff $X$ is discrete (where $X$ is a $T_{1}$-space): for the necessity construct for $z \approx x_{0}$ with $z \neq x_{0}$ a peak function. Since $X \backslash\left\{x_{0}\right\}$ is open we have ${ }^{*} x_{0} \notin \mu(z)$. But $f(z)={ }^{*} y_{1}$ and $f\left({ }^{*} x_{0}\right)={ }^{*} y_{0}$ so that $H$ is not evenly continuous.

Finally we consider general set-open topologies on subspaces $H$ of $C(X, Y)$. If $\alpha$ is a system of subsets of $X$ then we define $\alpha$ pts ${ }^{*} X:=\bigcup_{A \in \alpha}{ }^{*} A$ in analogy to the set of all compact points.

2.5. Proposition. Let $\alpha, \beta$ be systems of closed subsets and let $H$ be a completely regular family. If $\tau_{\alpha} \subset \tau_{\beta}$ on $H$ then $\alpha \mathrm{pts}^{*} X \subset \beta$ pts ${ }^{*} X$.

Proof. It is easy to see that $f \approx_{\alpha} \hat{y}_{0}$ iff $f(x) \approx y_{0}$ for all $x \in \alpha$ pts ${ }^{*} X$. Assume that there exists $z \in \alpha$ pts $^{*} X \backslash \beta$ pts $^{*} X$. A straightforward saturation argument shows that there exists $f \in{ }^{*} H$ with $f(x)={ }^{*} y_{0}$ for all $x \in \beta$ pts ${ }^{*} X$ and $f(z)={ }^{*} y_{1}$, in particular $f \approx_{\beta} \hat{y}_{0}$. Since $\tau_{\alpha} \subset \tau_{\beta}$ we obtain $f \approx_{\alpha} \hat{y}_{0}$, thus $f(z)={ }^{*} y_{1} \approx y_{0}$ yields the desired contradiction.

The converse in Proposition 2.5 is in general not true as a counterexample in [26] shows (even if $\beta$ is a network, thus Theorem 1.1 in [20] is not correct). This phenomenon depends on the fact that large sets $B \in \beta$ may induce trivial open sets $[B, V]$. In order to avoid this pathology it is sufficient to assume that $\beta$ is a "hereditary closed compact network" $[20$, p. 5$]$ : in this case one can prove an analogous formula as (2) replacing $\operatorname{cpt}^{*} X$ by $\beta$ pts $^{*} X$ and the converse of Proposition 2.5 has easily been proved. If in addition $Y$ is a uniform space then $\tau_{\beta}$ coincides with the topology of uniform convergence on the sets $B \in \beta$. As an illustration of Proposition 2.5 we mention that $\tau_{k}$ coincides with the topology of uniform convergence iff $\mathrm{cpt}^{*} X={ }^{*} X$ iff $X$ is compact; $\tau_{k}$ coincides with the topology $\tau_{p}$ of pointwise convergence iff $\mathrm{cpt}^{*} X={ }^{\sigma} X$ iff every compact set is finite. The topology of uniform convergence coincides with $\tau_{p}$ iff ${ }^{*} X={ }^{\sigma} X$ iff $X$ is finite.

\section{The AsColi Theorem}

A family $H \subset C(X, Y)$ is called pointwise bounded if for every $x \in X$ the orbit $\{f(x): f \in H\}$ is relatively compact and compactwise bounded if $\{f(x): f \in H, x \in K\}$ is relatively compact for every compact set $K \subset X$. The next theorem clarifies the relation between even continuity and relative compactness of subsets of $C(X, Y)$ with respect to an arbitrary topology $\tau$ on $C(X, Y)$. Surprisingly it turns out that condition (c) is the easiest to check for the compact-open topology. Condition (d) in Theorem 3.1 is explained in the next section and it is listed here only for completeness. The equivalence of $(a)$, (b), (d) is due to Noble (for Hausdorff spaces), the equivalence of (b) and (c) for the special case $\tau=\tau_{k}$ was proved in [18]. We note that (a) $\Rightarrow$ (b) in 
Theorem 3.1 is in general not true even if $Y$ is a compact $T_{1}$-space as simple counterexamples show; cf. [26].

3.1. Theorem. Let $Y$ be prehausdorff and $\tau$ be finer than the pointwise topology $\tau_{p}$. Then the following assertions are equivalent:

(a) The evaluation $e: H \times X \rightarrow Y$ is continuous for each $\tau$-compact set $H \subset C(X, Y)$.

(b) Every $\tau$-compact family $H \subset C(X, Y)$ is evenly continuous.

(c) The diagonal function $\Delta: X \rightarrow\left(C(Z, Y), \tau_{k}\right)$ defined by $\Delta(x)(f):=$ $f(x)$ is continuous where $Z:=(C(X, Y), \tau)$.

(d) $H$ is weakly conjoining.

Proof. (a) $\Rightarrow$ (b). Let $f \in{ }^{*} H$. Since $H$ is compact there exists $f_{0} \in H$ with $f \approx_{\tau} f_{0}$. The continuity of $e: H \times X \rightarrow Y$ yields $f(x) \approx f_{0}\left(x_{0}\right)$ for all $x \approx x_{0}$, in particular $f\left({ }^{*} x_{0}\right) \approx f_{0}\left(x_{0}\right)$. Let $y \in Y$ with $f\left({ }^{*} x_{0}\right) \approx y$. Formula (1) shows that ${ }^{*} f_{0}\left({ }^{*} x_{0}\right) \approx y$ and therefore $f(x) \approx y$. Thus $H$ is evenly continuous. (b) $\Rightarrow(\mathrm{a})$ is always valid: if $H$ is compact it is evenly continuous by $(b)$. Let $f \approx_{\tau} f_{0}$ and $x \approx x_{0}$. Since $\tau_{p} \subset \tau$ we have $f\left({ }^{*} x_{0}\right) \approx f_{0}\left(x_{0}\right)$ and the even continuity implies $f(x) \approx f_{0}\left(x_{0}\right)$. The equivalence of (a) and (c) is easy: $\Delta$ is continuous iff ${ }^{*} \Delta(x) \approx_{k} \Delta\left(x_{0}\right)$ for all $x \approx x_{0} \in X$. Since $Y$ is prehausdorff formula (2) is valid. Hence the last condition is equivalent to $f(x)={ }^{*} \Delta(x)(f) \approx \Delta\left(x_{0}\right)\left(f_{0}\right)=f_{0}\left(x_{0}\right)$ for all $f \in \mathrm{cpt}^{*} Z, f_{0} \in Z$ with $f \approx_{\tau} f_{0}$ and for all $x \approx x_{0} \in X$. But this is obviously equivalent to (a). The equivalence of $(b)$ and $(d)$ is proved in $\S 4$.

3.2. Theorem. The diagonal function $\Delta: X \rightarrow\left(C(Z, Y), \tau_{k}\right)$ with $Z=$ $\left(C(X, Y), \tau_{k}\right)$ is continuous if any of the following assertions is true:

(a) $X$ is a $k_{3}$-space and $Y$ regular.

(b) $X$ is a $k$-space and $Y$ prehausdorff.

Proof. It is known that $C(X, Y)$ is regular (prehausdorff) if $Y$ has this property. By our assumption at $X$ it suffices to show that $\Delta$ is continuous on each compact set $K \subset X$. But it is easy to see that this is equivalent to the following assertion (cf. the last proof): For all $f \in \mathrm{cpt}^{*} C(X, Y), f_{0} \in C(X, Y) f \approx_{\tau} f_{0}$ implies $f(x) \approx f_{0}\left(x_{0}\right)$ for all $x \in \operatorname{cpt}^{*} X, x_{0} \in X$ with $x \approx x_{0}$. If $\tau=\tau_{k}$, formula (2) shows the validity of this statement.

R. McCoy has claimed in [18, Corollary 1.6] that the diagonal function (in Theorem 3.2) is not continuous if $X$ is a $k_{Y}$-space but not a $k$-space. Theorem 3.2(a) shows that this is not true, e.g., let $Y=\mathbf{R}$ and $X=\mathbf{R}^{\mathbf{R}}$. His mistake depends on the implicit assumption that the space $k(X)$ (cf. the introduction) is completely regular (this fact was also used in Theorem 1.5 in [18]). The proof of Theorem 1.5 in [18] says essentially that for a completely regular $k_{Y}$-space the extension $k(X)$ is completely regular iff $X$ is a $k$-space.

3.3. Ascoli Theorem. Let $X$ be a $k$-space ( $k_{3}$-space resp.) and $Y$ be prehausdorff (regular resp.). Then the first two (all resp.) assertions are equivalent:

(a) $H \subset C(X, Y)$ is relatively compact for the compact-open topology.

(b) $H$ is evenly continuous and compactwise bounded.

(c) $H$ is evenly continuous and pointwise bounded. 
Proof. (a) $\Rightarrow$ (b). If the closure $\bar{H}$ is compact we know by Theorem 3.2 and Theorem 3.1 (c) $\Rightarrow($ a), (b) that $H$ is evenly continuous and $e: \bar{H} \times X \rightarrow Y$ continuous. Thus $H$ is also compactwise bounded. (b) $\Rightarrow(\mathrm{c})$ is trivial. We prove now (c) $\Rightarrow(\mathrm{a})$. In this case $C(X, Y)$ is regular hence it is enough to show that ${ }^{*} H \subset \mathrm{ns}^{*} C(X, Y)$. Since $H$ is pointwise bounded and evenly continuous every $f \in{ }^{*} H$ is ns-continuous. (4) shows that ${ }^{*} H \subset$ ns ${ }^{*} C(X, Y)$. Similarly we obtain for $(\mathrm{b}) \Rightarrow$ (a) that ${ }^{*} H \subset \mathrm{ns}{ }^{*} C(X, Y)$ using Proposition 2.2 and the compactwise boundedness of $H$. Consequently, if $H$ is closed then $H$ is compact. Thus it suffices to show that the pointwise closure $\bar{H}^{p}$ is also compactwise bounded and evenly continuous on compacta: let $K \subset X$ compact and choose $L$ compact and closed with $g(K) \subset L$ for all $g \in H$. Let $y \in Y$ and $V, V_{2}$ open neighborhoods of $y$ with $\bar{V}_{2} \cap L \subset V$. By the definition of even continuity there exists a neighborhood $U$ of $x_{0}$ and a neighborhood $W$ of $y$ such that $g(W) \subset V_{2}$ for all $g \in H$ with $g\left(x_{0}\right) \in W$. For $f \in \bar{H}^{p}$ there exists $g \in{ }^{*} H$ with $g\left({ }^{*} x\right) \approx f(x)$ for each $x \in X$. If $x \in U \cap K$ and $f\left(x_{0}\right) \in W$ then $g\left({ }^{*} x_{0}\right) \in{ }^{*} W$ and therefore $g\left({ }^{*} x\right) \in{ }^{*} V_{2} \cap{ }^{*} L$. Thus $f(U \cap K) \subset V$ and $f(K) \subset L$ for every $f \in \bar{H}^{p}$.

For a Hausdorff space $Y$ we obtain the following description: $H \subset C(X, Y)$ is compact iff $H$ is closed, evenly continuous and compactwise bounded. Our Example 2.4 shows that it is not enough to require only pointwise boundedness: the family $H:=\left\{f_{t}: t \in(0,1]\right\}$ is obviously pointwise bounded. Since every $f \in{ }^{*} H$ is ns-continuous $H$ is evenly continuous. Moreover it is easy to see that $H$ is closed in $C(X, Y)$ (but not in $F(X, Y)$ ). Furthermore, ${ }^{*} H$ is not contained in $\mathrm{ns}{ }^{*} C(X, Y)$ since for $t \approx 0$ the standard part function of ${ }^{*} f_{t}$ is not continuous. Thus $H$ is not relatively compact. For a treatment of the Ascoli Theorem for a large class of generalized uniform spaces we refer to [28].

\section{SPLitTING AND CONJOINING TOPOLOGIES}

Let $X, Y, T$ be topological spaces and let $f: T \times X \rightarrow Y$ be a function and define $f^{\mu}: T \rightarrow F(X, Y)$ by $f^{\mu}(t)(x):=f(t, x)$. Observe that the socalled exponential map $\mu$ is a bijection from $F(T \times X, Y)$ to $F(T, F(X, Y))$. A topology $\tau$ on $C(X, Y)$ is called splitting (weakly splitting) if $f^{\mu}: T \rightarrow$ $C(X, Y)$ is continuous for each continuous function $f: T \times X \rightarrow Y$ and for each (compact) space $T$. Conversely, $\tau$ is called conjoining (weakly conjoining) if $f: T \times X \rightarrow Y$ is continuous for every continuous function $f^{\mu}: T \rightarrow$ $C(X, Y)$ and each (compact) space $T$. For brevity we write $C_{\tau}(X, Y)$ instead of $(C(X, Y), \tau)$.

The next theorem generalizes some well-known facts for $C(X, Y)$ to arbitrary subspaces. Condition (c) says for $H=C(X, Y)$ that $\tau$ is conjoining. For the direction (a) $\Rightarrow$ (d) we know only references for the special case $\tau=\tau_{k}$.

4.1. Theorem. Let $\tau$ be a topology on $H \subset C(X, Y)$. Then the following assertions are equivalent:

(a) The evaluation $e: H \times X \rightarrow Y$ is continuous.

(b) $f \approx_{\tau} f_{0}$ implies $f(x) \approx f_{0}\left(x_{0}\right)$ for all $x \approx x_{0} \in X, f \in{ }^{*} H, f_{0} \in H$.

(c) For each space $T$ the continuity of $g^{\mu}: T \rightarrow H$ implies the continuity of $g: T \times X \rightarrow Y$. 
(d) For any prehausdorff space I the composition map $\circ: H \times C(I, X) \rightarrow$ $C(I, Y)(f, i) \mapsto f \circ i$ is continuous if $C(I, X)$ and $C(I, Y)$ are endowed with $\tau_{k}$.

Proof. (a) $\Leftrightarrow(b)$ is obvious and (b) $\Rightarrow(\mathrm{c})$ is straightforward. For $(\mathrm{c}) \Rightarrow(\mathrm{a})$ choose $T=H$ and observe that $e^{\mu}=\mathrm{id}: H \rightarrow H$ is continuous. For (d) $\Rightarrow$ (a) choose $I$ as a single set, then $C(I, X)=X, C(I, Y)=Y$ and $\circ$ is the evaluation. Let us prove $(b) \Rightarrow(d)$. Let $i \approx_{k} i_{0} \in C(I, X)$ and $f \approx_{\tau} f_{0} \in H$. By formula (2) we have to show that $f \circ i(t) \approx f_{0} \circ i_{0}\left(t_{0}\right)$ for all $t \in \operatorname{cpt}^{*} I$, $t_{0} \in I$ with $t \approx t_{0}$. But by formula $(2), i \approx_{k} i_{0}$ implies $i(t) \approx_{k} i_{0}\left(t_{0}\right)$ for all $t \in \operatorname{cpt}^{*} I, t_{0} \in I$ with $t \approx t_{0}$ and now apply (b).

4.2. Theorem. Let $\tau$ be a topology on $C(X, Y)$. Then the following assertions are equivalent:

(a) $f(x) \approx f_{0}\left(x_{0}\right)$ for all $x \approx x_{0} \in X$ implies $f \approx_{\tau} f_{0}$.

(b) $\tau$ is splitting.

(c) $\tau$ is coarser than every conjoining topology $\sigma$.

Proof. (a) $\Rightarrow$ (b) is left to the reader. (b) $\Rightarrow$ (c) is well known: since $e$ : $C_{\sigma}(X, Y) \times X \rightarrow Y$ is continuous (b) yields the continuity of $e^{\mu}=\mathrm{id}: C_{\sigma}(X, Y)$ $\rightarrow C_{\tau}(X, Y)$. For the nontrivial part (c) $\Rightarrow\left(\right.$ a) let $f \in{ }^{*} C(X, Y), f_{0} \in$ $C(X, Y)$ with $f(x) \approx f_{0}\left(x_{0}\right)$ be fixed. We construct a conjoining topology $\sigma$ (depending on $f, f_{0}$ ) with $f \approx_{\sigma} f_{0}$. Then (c) implies $f \approx_{\tau} f_{0}$ and the proof is finished. For every $g \in C(X, Y)$ with $g \neq f_{0}$ let $\{g\}$ be open. For $f_{0}$ we define neighborhoods $S_{U, V}:=\{g \in C(X, Y): g(U) \subset V\}$ where $U, V$ are open sets with $f_{0}(U) \subset V$ and $f\left({ }^{*} U\right) \subset{ }^{*} V$. Thus $f \in{ }^{*} S_{U, V}$ and therefore $f \approx_{\sigma} f_{0}$. It remains to show that $\sigma$ is conjoining. It suffices to prove 4.1(b). Let $g \approx_{\sigma} f_{0}$ and let $x \approx x_{0}$ and $V$ be an open neighborhood $V$ of $f_{0}\left(x_{0}\right)$. Since $m\left(x_{0}\right) \subset\left\{y \in{ }^{*} X: f(y) \in{ }^{*} V\right\}$ a saturation argument shows that there exists an open neighborhood $U$ of $x_{0}$ with $f\left({ }^{*} U\right) \subset{ }^{*} V$ and we can assume $f_{0}(U) \subset V$ by continuity of $f_{0}$. Since $g \approx_{\sigma} f_{0}$ we obtain $g \in{ }^{*} S_{U, V}$, equivalently, $g\left({ }^{*} U\right) \subset{ }^{*} V$. Thus $g(x) \in{ }^{*} V$ for every neighborhood of $f_{0}\left(x_{0}\right)$, therefore $g(x) \approx f_{0}\left(x_{0}\right)$.

4.3. Corollary. The finest splitting topology is the infimum of all conjoining topologies.

Proof. Theorem 4.2.

4.4. Remark. If $\tau$ is only coarser than every Hausdorff conjoining topology we can infer (a) of Theorem 4.2 if $f \in{ }^{*} H \backslash{ }^{\sigma} H$ : consider neighborhoods of the form $S_{U, V} \backslash\{h\}$ with $U, V$ as above and $h \in H \backslash\left\{f_{0}\right\}$. Then $\sigma$ is a regular Hausdorff space. Observe that every conjoining topology $\sigma$ is Hausdorff if $Y$ is Hausdorff since by Theorem $4.1 \sigma$ contains the compact-open topology (cf. formula (2)).

Let us consider the compact-open topology: since the direction " $\Leftarrow$ " in (2) is valid without any separation assumption Theorem 4.2 (a) $\Rightarrow$ (b) shows that $\tau_{k}$ is always a splitting topology (Theorem 2.5.2 in [20]). Thus the exponential map $\mu$ maps $C(T \times X, Y)$ into $C\left(T, C_{\tau_{k}}(X, Y)\right)$. If the characterization (2) is valid for the spaces $C(X, Y), C(T, C(X, Y))$ and $C(T \times X, Y)$ then it is straightforward to verify that $\mu$ is actually an embedding. Note that $C(X, Y)$ is prehausdorff if $Y$ is prehausdorff. This shows the first part of 
4.5. Theorem. Let $Y$ be prehausdorff or let $X$ and $T$ be prehausdorff. Then the exponential map $\mu: C(T \times X, Y) \rightarrow C\left(T, C_{\tau_{k}}(X, Y)\right)$ is an embedding. It is a homeomorphism if $T \times X$ is a $k_{Y}$-space or $X$ is locally compact.

Proof. Let $f: T \times X \rightarrow Y$ be a function such that $f^{\mu}: T \rightarrow C(X, Y)$ is continuous, i.e., ${ }^{*} f^{\mu}(t) \approx f^{\mu}\left(t_{0}\right)$ for all $t \approx t_{0} \in T$. By (2) we obtain ${ }^{*} f(x, t) \approx$ $f_{0}\left(t_{0}, x_{0}\right)$ for all $x \in \mathrm{cpt}^{*} X$ with $x \approx x_{0}$ and $t \approx t_{0}$, in particular $f$ is continuous on each compact set of $T \times X$. If $T \times X$ is a $k_{Y}$-space we infer the continuity of $f$. If $X$ is locally compact we have $\operatorname{cpt}^{*} X=\mathrm{ns}^{*} X$ and the continuity of $f$ is obvious.

If $X$ is locally compact then $\mu$ is bijective for any space $T$, thus $\tau_{k}$ is conjoining; cf. also Theorem 2.1 and Theorem 4.1. If $H=C(X, Y)$ is a completely regular family then $\tau_{k}$ is conjoining iff $X$ is locally compact: for the necessity choose for $z \in \mathrm{ns}^{*} X \backslash \mathrm{cpt}^{*} X$ a peak function $f \in{ }^{*} H$ with $f(z)=$ ${ }^{*} y_{1}$ and $f \approx_{k} \hat{y}_{0}$. Since $4.1(\mathrm{~b})$ is not satisfied we obtain a contradiction. More generally, Arens has proved that the existence of a smallest conjoining (= conjoining and splitting) topology implies the local compactness. We refer to [16] for a detailed discussion of the exponential law for spaces with very weak separation properties and further generalizations.

Now let us prove the equivalence of $(\mathrm{b})$ and (d) in Theorem 3.1: let $t \approx t_{0} \in T$ and $x \approx x_{0} \in X$. It suffices to show that ${ }^{*} f(x, t) \approx f\left(t_{0}, x_{0}\right)$. By assumption we have ${ }^{*} f^{\mu}(t) \approx f^{\mu}\left(t_{0}\right)$. But $\tau$ is finer than the pointwise topology thus ${ }^{*} f^{\mu}(t)\left({ }^{*} x_{0}\right) \approx f^{\mu}\left(t_{0}\right)\left(x_{0}\right)=f\left(t_{0}, x_{0}\right)=: y$. Moreover $f^{\mu}(T)$ is compact and by

(b) evenly continuous. Hence ${ }^{*} f^{\mu}(t)(x) \approx y$, i.e., ${ }^{*} f(x, t) \approx f\left(t_{0}, x_{0}\right)$. For (d) $\Rightarrow$ (a) observe that $e^{\mu}=\mathrm{id}: H \rightarrow C(X, Y)$ is obviously continuous.

\section{APPlications to HYPERSPACES}

Let $\chi_{A}: X \rightarrow\{0,1\}$ be the characteristic function of a subset $A$ of $X$. Then $\chi: \mathscr{P}_{\mathrm{cl}}(X) \rightarrow C(X,\{0,1\})$ defined by $A \mapsto \chi_{A}$ is a bijection if $Y=$ $\{0,1\}$ is endowed with the Sierpinski topology $\{\varnothing,\{0\}, Y\}$. The compactopen topology on $\mathscr{P}_{\mathrm{cl}}(X)$ is generated by the sets $\left\{A \in \mathscr{P}_{\mathrm{cl}}(X): \chi_{A}(K) \subset\{0\}\right\}=$ $\{A: A \subset X \backslash K\}$ where $K$ is an arbitrary compact set. If $X$ is prehausdorff then formula (2) shows that $A \approx_{k} A_{0} \in \mathscr{P}_{\mathrm{cl}}(x)$ iff $\chi_{A}(x) \approx \chi_{A_{0}}\left(x_{0}\right)$ for all $x \in \mathrm{cpt}^{*} X, x_{0} \in X$ with $x \approx x_{0}$ iff $\operatorname{st}\left(A \cap \mathrm{cpt}^{*} X\right) \subset A_{0}$ where st $A:=\{x \in$ $X: \exists y \in A$ with $y \approx x\}$. The topology $\tau_{+}\left(\tau_{-}\right.$resp.) generated by the sets $U^{+}:=\{A: A \subset U\} \quad\left(U^{-}:=\{A: A \cap U \neq \varnothing\}\right)$ is called the upper (lower resp.) semifinite topology. It is easy to see that $A \approx_{-} A_{0} \in \mathscr{P}_{\mathrm{cl}}(A)$ iff $\operatorname{st} A \supset A_{0}$. The supremum $\tau_{F}$ of $\tau_{k}$ and $\tau_{-}\left(\tau_{V}\right.$ of $\tau_{+}$and $\tau_{-}$resp.) is called the Fell topology (Vietoris topology). It is not very difficult to see that $\left(\mathscr{P}_{\mathrm{cl}}(X), \tau_{F}\right)$ is always a compact $T_{1}$-space (use the proof of Proposition I.4 in [33] and observe that the direction " $\Leftarrow$ " in formula (2) is always valid). The above remarks show that

$$
A \approx_{F} A_{0} \Leftrightarrow \operatorname{st}\left(A \cap \mathrm{cpt}^{*} X\right) \subset A_{0} \subset \mathrm{st} A
$$

if $X$ is prehausdorff or basic locally compact. In the latter case we have $A \approx_{F}$ $A_{0}$ iff st $A=A_{0}$, and $\mathscr{P}_{\mathrm{cl}}(X)$ is a (compact) Hausdorff space. These results were proved in [7] and [33] (where $\tau_{F}$ is called the $S$-compact-topology).

Narens has introduced the $N$-compact topology $\tau_{N}$ : a set $H \subset \mathscr{P}_{\mathrm{cl}}(X)$ is $\tau_{N}$-open if $k_{N}\left(A_{0}\right):=\left\{A \in{ }^{*} \mathscr{P}_{\mathrm{cl}}(X):\right.$ st $\left.A=A_{0}\right\} \subset{ }^{*} H$ for every $A_{0} \in H$. The 
$N$-compact topology is the topology generated by the pseudomonads $k_{N}\left(A_{0}\right)$ and is therefore the finest of the topologies $\tau$ with the property that st $A=A_{0}$ implies $A \approx_{\tau} A_{0}$, in particular $\tau_{F} \subset \tau_{N}$. Observe that $\tau_{N}=\tau_{F}$ if $X$ is basic locally compact. In this section we show that $\tau_{N}$ is identical with the convergence topology (for definition see below) which was already discussed by many authors, see e.g. [8]. But at first we need the following characterization:

5.1. Proposition. The $N$-compact topology is the infimum of all conjoining topologies $\sigma$ with $\tau_{-} \subset \sigma$.

Proof. Let $\sigma$ be conjoining with $\tau_{-} \subset \sigma$. Theorem 4.1 shows that $A \approx_{\sigma} A_{0}$ implies $\chi_{A}(x) \approx \chi_{A_{0}}\left(x_{0}\right)$ for all $x \approx x_{0}$, or equivalently, st $A \subset A_{0}$. Since $\tau_{-} \subset \sigma$ we have st $A \supset A_{0}$. Hence st $A=A_{0}$ and therefore $A \approx_{N} A_{0}$. This shows $\tau_{N} \subset \sigma$. For the other inclusion let $\tau$ be the infimum of all conjoining topologies containing $\tau_{-}$. Then $\tau \subset \tau_{N}$ if we can prove that st $A=A_{0}$ implies $A \approx_{\tau} A_{0}$. Define a conjoining topology $\sigma$ (depending on $A, A_{0}$ ) as in the proof of Theorem 4.2 and let $\rho$ be the supremum of $\sigma$ and $\tau_{-}$. Then $\tau \subset \rho$ and by construction $A \approx_{\sigma} A_{0}$. Now st $A=A_{0}$ implies $A \approx_{\rho} A_{0}$ and $A \approx_{\tau} A_{0}$.

For the definition of the convergence topology we need some terminology: for a net $\left(A_{i}\right)_{i \in I}$ in the hyperspace $\lim \inf A_{i} \quad\left(\lim \sup A_{i}\right.$ resp.) denotes the set of all $x \in X$ such that for every neighborhood $V$ of $x V \cap A_{i} \neq \varnothing$ for almost all (for infinitely many) $i \in I$. If the index set $I$ is an element of the standard universe ${ }^{*} I_{\infty}$ denotes the set of all $i \in{ }^{*} I$ with $i \geq{ }^{*} i_{0}$ for all $i_{0} \in I$. It is not very hard to see that

$$
\liminf A_{i}=\bigcap_{i \in *_{\infty}} \mathrm{st}^{*} A_{i} \subset \bigcup_{i \in I_{\infty}} \mathrm{st}^{*} A_{i}=\lim \sup A_{i} .
$$

$A_{0}$ is the limit of $\left(A_{i}\right)_{i \in I}$ if $A_{0}=\liminf A_{i}=\limsup A_{i}$, or equivalently, $A_{0}=\mathrm{st}^{*} A_{i}$ for all $i \in{ }^{*} I_{\infty}$. A subset $H \subset \mathscr{P}_{\mathrm{cl}}(X)$ is closed in the convergence topology $\tau_{C}$ if for every $A_{0} \in \mathscr{P}_{\mathrm{cl}}(X)$ and every net $\left(A_{i}\right)_{i \in I}$ the validity of $\lim A_{i}=A_{0}$ implies $A_{0} \in H$. It is not very difficult to see that one can require that the index set $I$ in the above definition is a set in the standard universe.

5.2. Theorem. The N-compact topology coincides with the convergence topology. It contains the supremum of the finest splitting topology and $\tau_{-}$.

Proof. We show $\tau_{N} \subset \tau_{C}$. Let $H$ be $\tau_{N}$-closed and $\left(A_{i}\right)_{i \in I}$ be a net in $H$ with $\lim A_{i}=A_{0}$. Since $I$ can be assumed to be in the standard universe (7) yields st ${ }^{*} A_{i}=A_{0}$ for all $i \in{ }^{*} I_{\infty}$. Thus st ${ }^{*} A_{i} \approx_{N} A_{0}$ and by $\tau_{N}$-closedness of $H$ we infer $A_{0} \in H$. Thus $H$ is $\tau_{C}$-closed. For the other inclusion it suffices to show that $\tau_{C}$ is smaller than every conjoining topology $\sigma$ containing $\tau_{-}$. Let $H$ be $\tau_{C}$-closed, we show that it is $\sigma$-closed: let $A_{0}$ be in the $\sigma$-closure of $H$ and $\left(A_{i}\right)_{i \in I}$ a net in $H$ converging in the $\sigma$-topology to $A_{0}$. Then st ${ }^{*} A_{i}=A_{0}$ for every $i \in{ }^{*} I_{\infty}$. (7) yields $\lim A_{i}=A_{0}$. Thus $A_{0} \in H$. This proves the first statement. Let $\rho$ be the finest splitting topology; then st $A \subset A_{0}$ implies $A \approx_{\rho} A_{0}$ and this yields $\rho \subset \tau_{N}$.

Our next theorem answers a question in [3, p. 53] positively for the class of all topological vector spaces; our proof depends on arguments given by Wattenberg for the special case of the rationals $\mathbf{Q}$. $\mathbf{Z}$ denotes the set of all integers. 
5.3. Theorem. Let $X$ be a topological vector space over $\mathbf{Q}$. Then the Fell topology and the convergence topology coincide iff $X$ is locally compact.

Proof. We only have to show that $\tau_{F}=\tau_{C}$ implies the local compactness. Observe that $\mathbf{Z} x:=\{n x: n \in \mathbf{Z}\}$ is closed for every $x \in X$. It is easy to see that the set $\mathscr{S}$ of all closed subgroups is $\tau_{C}$-closed; cf. [24]. If $X$ is not locally compact there exists $x_{0} \in X, x \in{ }^{*} X$ such that $x \approx x_{0}$ and $x \notin \mathrm{cpt}^{*} X$. We can assume that $x_{0}$ is not zero. Then $A:={ }^{*} \mathbf{Z} x \in{ }^{*} \mathscr{S}$. Moreover $I:=\left\{n \in \mathbf{Z}: n \cdot x \in \operatorname{cpt}^{*} X\right\}$ is an ideal of $\mathbf{Z}$ with $-1 \notin I$. Obviously $I \cdot x_{0}$ and $A_{0}:=I \cdot x_{0} \cup\left\{x_{0}\right\}$ are closed. It is not very difficult to see that $\operatorname{st}\left(A \cap \operatorname{cpt}^{*} X\right) \subset A_{0} \subset \mathrm{st} A$. (6) yields $A \approx_{F} A_{0}$. But $A_{0}$ is not a subgroup. Therefore $\mathscr{S}$ is not $\tau_{F}$-closed.

The next theorem answers a question in [33] negatively. As usual $X_{\infty}:=$ $X \cup\{\infty\}$ denotes the Alexandroff compactification of a topological space $X$. If $X$ is a $T_{1}$-space we call $j: X_{\infty} \rightarrow \mathscr{P}_{\mathrm{cl}}(X)$ defined by $j(x)=\{x\}$ and $j(\infty)=\varnothing$ the natural injection.

5.4. Theorem. Let $X$ be a topological vector space over $\mathbf{Q}$. Then the union map $\cup: \mathscr{P}_{\mathrm{cl}}(X) \times \mathscr{P}_{\mathrm{cl}}(X) \rightarrow \mathscr{P}_{\mathrm{cl}}(X)$ defined by $\cup(A, B):=A \cup B$ is continuous for $\tau_{C}$ iff $X$ is locally compact.

Proof. If $X$ is locally compact and regular then $\tau_{F}=\tau_{C}$. But it is easy to see that $U$ is always continuous for the Fell topology. For the converse let $x, x_{0}$ be as in the last proof. Clearly $B:=\left\{n \cdot x: n \in{ }^{*} Z, n \neq 1\right\}$ is in ${ }^{*} \mathscr{P}_{\mathrm{cl}}(X)$. Since st $B=\mathbf{Z} x_{0} \backslash\left\{x_{0}\right\}=: C$ we have trivially $B \approx_{N} C$. On the other side $A:=\{x\} \approx_{N} \varnothing$ since the natural injection $j: X_{\infty} \rightarrow \mathscr{P}_{\mathrm{cl}}(X)$ is an embedding; cf. [33]. If $\cup$ is continuous then $A \cup B \approx_{N} C$. But $C$ is not a subgroup and the set of all subgroups is $\tau_{C}$-closed. Thus $A \cup B$ cannot be a ${ }^{*}$-subgroup, a contradiction to $A \cup B={ }^{*} \mathrm{Z} x$.

Obviously every conjoining topology $\sigma$ containing $\tau_{-}$is Hausdorff. So it is natural to ask whether $\tau_{N}$ is coarser than all conjoining Hausdorff topologies. Surprisingly the following is true:

5.5. Theorem. Let $X$ be a Hausdorff space. Then the convergence topology is coarser than all conjoining Hausdorff topologies if and only if $X$ is discrete and finite.

Proof. If $X$ is nondiscrete there exists $x_{0} \in X$ and $x \in{ }^{*} X$ with $x \approx x_{0}$ and $x \neq{ }^{*} x_{0}$. Choose $x_{1} \in X \backslash\left\{x_{0}\right\}$. Then $A:=\{x\} \in{ }^{*} \mathscr{P}_{\mathrm{cl}}(X)$ and $A_{0}:=$ $\left\{x_{0}, x_{1}\right\} \in \mathscr{P}_{\mathrm{cl}}(x)$ and we have st $A \subset A_{0}$, st $A \neq A_{0}$ and $A \notin \mathscr{P}_{\mathrm{cl}}(X)$. As pointed out in Remark 4.4 there exists a conjoining Hausdorff topology $\sigma$ with $A \approx_{\sigma} A_{0}$. Since st $A=\left\{x_{0}\right\} \neq A_{0} \sigma$ cannot contain $\tau_{-}$, in particular not $\tau_{N}$. This contradicts our assumption. Similarly, if $X$ is nonfinite there exists $x \in{ }^{*} X \backslash \mathrm{ns}^{*} X$. Now choose $A=\{x\}$ and $A_{0}=\left\{x_{0}\right\}$ for some $x_{0} \in X$. For the converse observe that ${ }^{*} H={ }^{\sigma} H$ for every subset $H \subset \mathscr{P}_{\mathrm{cl}}(X)$. Thus the monads with respect to $\sigma$ contains only standard sets. But since $\sigma$ is Hausdorff we can infer that $\sigma$ is discrete and $\tau_{N} \subset \sigma$ is trivial.

Arens and Dugundji have conjectured in [2, p. 29] that the Vietoris topology is the smallest conjoining Hausdorff topology if $X$ is a compact Hausdorff space. But this is not true unless $X$ is discrete since $\tau_{V}=\tau_{N}$ for a compact regular space. Now let us compare $\tau_{V}$ and $\tau_{N}$. It is not very difficult to see 
that $\tau_{N} \subset \tau_{V}$ if $X$ is regular. Narens has shown that $\varnothing$ is an isolated point of $\left(\mathscr{P}_{\mathrm{cl}}(X), \tau_{N}\right)$ iff $X$ is compact. It follows immediately that $X$ is compact iff $\tau_{V} \subset \tau_{N}$ iff $\tau_{V} \subset \tau_{F}$.

5.6. Theorem. Let $X$ be a Hausdorff space. Then the following assertions are equivalent:

(a) $X$ is locally compact.

(b) There exists a topology $\tau$ on $\mathscr{P}_{\mathrm{cl}}(X)$ with: $A \approx_{\tau} A_{0} \Leftrightarrow$ st $A \subset A_{0}$.

(c) There exists a topology $\tau$ on $\mathscr{P}_{\mathrm{cl}}(X)$ with: $A \approx_{\tau} A_{0} \Leftrightarrow \mathrm{st} A=A_{0}$.

(d) $A \approx_{N} A_{0} \Leftrightarrow$ st $A=A_{0}$.

(e) $A \approx_{F} A_{0} \Leftrightarrow$ st $A=A_{0}$.

(f) $\mathscr{P}_{\mathrm{cl}}(X)$ is a (compact) Hausdorff space for $\tau_{F}$ or $\tau_{C}$.

(g) $e: \mathscr{P}_{\mathrm{cl}}(x) \times X \rightarrow\{0,1\}$ is continuous for $\tau_{k}, \tau_{F}$ or $\tau_{C}$.

(h) $\mathscr{P}_{\mathrm{cl}}(X) \times X$ is a $k$-space for $\tau_{k}, \tau_{F}$ or $\tau_{C}$.

Proof. For (a) $\Rightarrow$ (b) choose $\tau=\tau_{k}$ and for (b) $\Rightarrow$ (c) as the supremum of the topology in (b) and $\tau_{-}$. For (c) $\Rightarrow(\mathrm{d})$ observe that every $\tau$-open set is $\tau_{N}$-open, i.e., $\tau \subset \tau_{N}$. Thus $A \approx_{N} A_{0}$ implies $A \approx_{\tau} A_{0}$ and by (c) st $A=A_{0}$. (d) $\Rightarrow(\mathrm{f})$ and (a) $\Rightarrow$ (e) $\Rightarrow$ (f) are clear. For (f) $\Rightarrow$ (a) consider the natural embedding $j: X_{\infty} \rightarrow \mathscr{P}_{\mathrm{cl}}(X)$. Thus (a)-(f) are equivalent. Theorem 2.1 yields (a) $\Rightarrow(\mathrm{g})$. We show now $(\mathrm{g}) \Rightarrow(\mathrm{d})$. We can assume that $e$ is continuous for $\tau_{N}$. Then $x \approx x_{0}$ and $A \approx \approx_{N} A_{0}$ implies $\chi_{A}(x) \approx \chi_{A_{0}}\left(x_{0}\right)$, in other words st $A \subset A_{0}$. Since $\tau_{-} \subset \tau_{N}$ we obtain st $A=A_{0}$. (a) $\Rightarrow$ (h) is clear since $X \times \mathscr{P}_{\mathrm{cl}}(X)$ is locally compact. For the last implication $(\mathrm{h}) \Rightarrow(\mathrm{g})$ it is enough to show that $e$ is continuous on every compact set $K \times \mathscr{P}_{\mathrm{cl}}(X)$. Let $x \in{ }^{*} K$, $x_{0} \in K$ with $x \approx x_{0}$ and $A \approx_{\tau} A_{0}$ (where $\tau=\tau_{k}, \tau_{F}$ or $\tau_{C}$ ). We have to show that ${ }^{*} e(A, x)={ }^{*} \chi_{A}(X) \approx \chi_{A_{0}}\left(x_{0}\right)=e\left(A_{0}, x_{0}\right)$, or equivalently, $A \approx_{\tau} A_{0}$ implies $\operatorname{st}\left(A \cap \mathrm{cpt}^{*} X\right) \subset A_{0}$. Since $\tau \supset \tau_{k}$ this is true.

5.7. Theorem. Let $X$ be a locally compact non-Hausdorff space such that every compact set is closed. Then the evaluation $e: C(X,\{0,1\}) \times X \rightarrow\{0,1\}$ is not continuous for $\tau_{k}$ or $\tau_{F}$, and $\mathscr{P}_{\mathrm{cl}}(X)$ is not a Hausdorff space for the Fell topology.

Proof. Using the proof of $(\mathrm{g}) \Rightarrow$ (d) $\Rightarrow$ (f) of the last theorem we infer that $\mathscr{P}_{\mathrm{cl}}(X)$ is a Hausdorff space for $\tau_{F}$. Since every compact set is closed it is easy to see that $j: X_{\infty} \rightarrow \mathscr{P}_{\mathrm{cl}}(X)$ is continuous, a contradiction to the fact, that $X$ is non-Hausdorff.

Recall that $X$ is an $A 2$-space if it has a countable base for the topology. $X$ has a point-countable base if there exists a base $\mathscr{B}$ for the topology such that $\mathscr{B}_{x}:=\{B \in \mathscr{B}: x \in B\}$ is countable. It is easy to see that $X$ is an $A 2$-space iff $X$ is separable with a point-countable base. A regular space is cosmic if it is a continuous image of a separable metric space; $c f$. [21].

5.8. Theorem. Let $X$ be a Hausdorff space and let $\mathscr{P}_{\mathrm{cl}}(X)$ be endowed with $\tau_{F}$ or $\tau_{C}$. Then the following assertions are equivalent:

(a) $\mathscr{P}_{\mathrm{cl}}(X)$ is metrizable.

(b) $\mathscr{P}_{\mathrm{cl}}(X)$ is an A2-space.

(c) $\mathscr{P}_{\mathrm{cl}}(X)$ possesses a point-countable base.

(d) $X$ is a locally compact A2-space. 
If $X$ is metrizable or a cosmic space then the following assertion is equivalent:

(e) $\mathscr{P}_{\mathrm{cl}}(X)$ is an A1-space.

Proof. Since $\mathscr{P}_{\mathrm{cl}}(X)$ is always compact we have (a) $\Rightarrow(\mathrm{b}) \Rightarrow(\mathrm{c})$. Now let us prove (c) $\Rightarrow(\mathrm{d})$ and (e) $\Rightarrow(\mathrm{d})$. Since $X_{\infty}$ is a subspace of $\mathscr{P}_{\mathrm{cl}}(X)$ we know that $X_{\infty}$ and $X_{\infty} \times \mathscr{P}_{\mathrm{cl}}(X)$ are $A 1$-spaces. Thus $X_{\infty} \times \mathscr{P}_{\mathrm{cl}}(X)$ is a $k$-space and by Theorem $5.6(\mathrm{~h}) \Rightarrow$ (a), $X$ is a locally compact, $\sigma$-compact $A 1$-space. In case (c), $X_{\infty}$ is even a compact Hausdorff space with a point-countable base. Proposition 2.1 in [4] shows that $X_{\infty}$ is an $A 2$-space. If $X$ is metrizable, well-known results yield (d). If $X$ is cosmic and locally compact then $X$ is metrizable; cf. [21, p. 994]. For (d) $\Rightarrow$ (a) we refer to [8].

Observe that every condition in Theorem 5.8 implies $\tau_{F}=\tau_{C}$. Moreover it is not very difficult to show that every closed subset of $X$ is necessarily separable if $\left(\mathscr{P}_{\text {cl }}(X), \tau_{F}\right)$ is an $A 1$-space using the proof of the following result in [34]: the space $\mathscr{P}_{\text {co }}(X)$ of all compact subsets is an $A 1$-space for the Vietoris topology iff (i) every compact subset is separable and (ii) every compact set has a countable neighborhood base. Smithson has shown that $\mathscr{P}_{\text {co }}(X)$ is an $A 1$-space for $\tau_{V}$ iff $X$ is "compactly second countable"; cf. [30]. Smithson has asked in [30] under what conditions $X$ is compactly second countable if (a) all compact sets are $A 2$-spaces or if (b) every compact set has a countable neighborhood base. Obviously the results of [34] give reasonable conditions. The results in [22] yield the following characterization: If a regular space $X$ satisfies $(a)$ then $\mathscr{P}_{\text {co }}(X)$ is an $A 1$-space for $\tau_{V}$ iff $X$ is a compact-covering open image of a metric space.

\section{REALCOMPACT SPACES}

In this section we give a nonstandard characterization of real compactness. Our results are complementary to those in [5] and [32]. As usual $C^{b}(X)$ denotes the set of all continuous, real-valued and bounded functions. Instead of $C(X, \mathbf{R})$ we write $C(X)$.

Definition. Let $H \subset C(X, Y)$. Then $H$-ns ${ }^{*} X:=\left\{x \in{ }^{*} X:{ }^{*} f(x) \in \mathrm{ns}^{*} Y\right.$ for all $f \in H\}$ is called the set of all $H$-nearstandard points. In case of $H=C(X)$ we write psns ${ }^{*} X$ instead of $C(X)$-ns ${ }^{*} X$ and call it the set of all pseudonearstandard points.

A topological space is pseudocompact if every continuous real-valued function is bounded. It is easy to see that $X$ is pseudocompact iff ${ }^{*} X=$ psns $^{*} X$, an analogy of the nonstandard characterization of compactness.

Let $H$ be $C(X)$ or $C^{b}(X), I$ be an ideal of $H$, and let $p: H \rightarrow H / I$ be the natural projection. Recall that the image of the set of all constant functions is a subfield of $H / I$ which can be identified with $\mathbf{R}$. If this subfield is the whole space $H / I$ then $I$ is called real. A maximal ideal is free if it is not of the form $\{f \in H: f(x)=0\}$ for some $x \in X$. A completely regular Hausdorff space $X$ is real compact if every real maximal ideal $M$ of $C(X)$ is of the form $\{f \in C(X): f(x)=0\}$ for some $x \in X$.

6.1. Proposition. Let $H=C(X)$ or $H=C^{b}(X)$. Then $\left\{f \in H:{ }^{*} f(x) \approx 0\right\}$ is a real, maximal ideal for every $x \in H$-ns ${ }^{*} X$.

Proof. Since $x \in H$-ns ${ }^{*} X$ the map st $_{x}: H \rightarrow \mathbf{R}$ defined by $\mathbf{s t}_{x} f:={ }^{*} f(x)$ is well defined and obviously a surjective homomorphism. 
6.2. Example. Let $H=C(X)$. Then $H_{x}:=\{f \in H: f(x) \approx 0\}$ is an ideal iff $x \in$ psns ${ }^{*} X$ : if $x \notin$ psns ${ }^{*} X$ then there exists $g \in H$ with ${ }^{*} g(x) \in{ }^{*} \mathbf{R} \backslash$ ns $^{*} \mathbf{R}$. We can assume that $g$ is invertible since $H$ contains the maximum of $g$ and a constant function. If $H_{x}$ is an ideal then $1=g \cdot g^{-1} \in H_{x}$ yields the desired contradiction.

6.3. Theorem. Let $M$ be an ideal of $H=C(X)$ or $H=C^{b}(X)$. Then there exists $x \in{ }^{*} X$ such that $M \subset\{f \in H: f(x) \approx 0\}=: H_{x}$. In this case the following assertions are equivalent:

(a) $M$ is a real maximal ideal.

(b) $x \in H$-ns ${ }^{*} H$ and $M$ is a maximal ideal.

(c) $M=H_{x}$.

Proof. For the first statement cf. the proof of Theorem 2.1 in [5]. For (a) $\Rightarrow$ (b) let $f \in H$ and $M$ be a real maximal ideal. Then there exists a constant function $r \in H$ and $g \in M$ with $g+f=r$. Since $M \subset H_{x}$ we have ${ }^{*} g(x) \approx 0$, hence ${ }^{*} f(x) \in \mathrm{ns}^{*} X$. This shows $x \in H$-ns ${ }^{*} X$. For (b) $\Rightarrow(\mathrm{c}) \Rightarrow$ (a) use Proposition 6.1, the maximality of $M$ and Example 6.2.

6.4. Theorem. Let $X$ be a completely regular Hausdorff space. Then $\mathrm{ns}^{*} X=$ psns ${ }^{*} X$ if and only if $X$ is real compact.

Proof. Let $M$ be a real maximal ideal. Thus there exists $x \in$ psns $^{*} X=\mathrm{ns}^{*} X$ with $M=\left\{f \in C(X):{ }^{*} f(x) \approx 0\right\}$. Choose $x_{0} \in X$ with $x \approx x_{0}$. Then $M=$ $\left\{f \in C(X): f\left(x_{0}\right)=0\right\}$ and $X$ is real compact. Conversely, let $x \in$ psns $^{*} X$. Then $M:=\left\{f \in C(X):{ }^{*} f(x) \approx 0\right\}$ is a real maximal ideal, hence of the form $\left\{f \in C(X): f\left(x_{0}\right)=0\right\}$ for some $x_{0} \in X$ by real compactness. It suffices to show that $x \approx x_{0}$. Assume that there exists an open neighborhood $U$ of $x_{0}$ with $x \notin{ }^{*} U$. Choose $f \in C(X)$ with $f(X \backslash U)=\{1\}$ and $f\left(x_{0}\right)=0$. Then $f$ is in $\left\{f \in C(X): f\left(x_{0}\right)=0\right\}$ but $f \notin M$, a contradiction.

Theorem 6.3 and Proposition 6.1 show that the real maximal ideals of $C(X)$ are of the form $\left\{f \in C(X):{ }^{*} f(x) \approx 0\right\}$ with $x \in$ psns ${ }^{*} X$. For a nonstandard description of an arbitrary maximal ideal we refer to [5]. The following wellknown result is an obvious consequence of Theorem 6.4: A completely regular Hausdorff space $X$ is compact if and only if it is real compact and pseudocompact.

6.5. Proposition. A closed subset of a real compact space is real compact.

Proof. Let $x \in$ psns $^{*} A$. Then $x \in$ psns $^{*} X \subset$ ns $^{*} X$. Since $A$ is closed we obtain $x \in \mathrm{ns}^{*} A$.

6.6. Theorem. Let $X$ be a completely regular Hausdorff space and $S \subset X$. Then psns* $(S \cup K)=$ psns $^{*} S \cup \mathrm{ns}^{*}(S \cup K)$ for every compact subset $K \subset X$. In particular: if $S$ is real compact (pseudocompact) then $S \cup K$ is real compact (pseudocompact).

Proof. Let $y \in$ psns $^{*}(S \cup K)$. Assume that $y \notin \mathrm{ns}^{*}(S \cup K)$. Then $y \notin$ $\bigcup_{x \in K} m(x)=\bigcap_{U \in \tau, K \subset U}{ }^{*} U \supset{ }^{*} K$. Thus there exists a continuous function $g: X \rightarrow \mathbf{R}$, disjoint open sets $U, V$ with $K \subset U$ and $y \in{ }^{*} V$ such that $g(U)=\{0\}$ and $g(V)=\{1\}$. Now let $f: S \rightarrow \mathbf{R}$ be continuous. It suffices to show that ${ }^{*} f(y) \in$ ns ${ }^{*} \mathbf{R}$. It is not very difficult to see that $\tilde{f}: S \cup K \rightarrow \mathbf{R}$ defined by $\tilde{f}(x)=f(x) g(x)$ for $x \in S$ and $\tilde{f}(x)=0$ otherwise is a continuous 
function. Since $y \in$ psns ${ }^{*}(S \cup K)$ we infer that ${ }^{*} f(y)={ }^{*} f(y){ }^{*} g(y)={ }^{*} \tilde{f}(y) \in$ $n{ }^{*} \mathbf{R}$. The other inclusion is trivial.

For $x, y \in{ }^{*} X$ define $y \approx_{\beta} x$ to mean that ${ }^{*} f(y) \approx{ }^{*} f(x)$ for all $f \in$ $C^{b}(X)$. It is well known that $\beta X:={ }^{*} X / \approx_{\beta}$ is the Stone-Čech compactification of $X$. We want to construct the Hewitt real compactification $v X$. Define $v X:=$ psns $^{*} X / \approx_{\beta} \subset \beta X$ with the relative topology. We have to show that $v X$ is a real compact space such that $X$ is dense and $C$-embedded in $v X$. Clearly $X$ is dense in $v X$ since $X$ is dense in $\beta X$. Let $f: X \rightarrow \mathbf{R}$ be continuous. Then $f^{v}: v X \rightarrow \mathbf{R}$ defined by $f^{v}([x]):=$ st $^{*} f(x)$ with $x \in$ psns $^{*} X$ is a continuous (well-defined) extension. Thus $X$ is $C$-embedded in $v X$ and the restriction map $R: C(v X) \rightarrow C(X)$ is a bijective algebra homomorphism. Let us show that $v X$ is real compact: let $M \subset C(v X)$ be a real maximal ideal. Then $R(M)$ is a real maximal ideal and hence of the form $\left\{g \in C(X):{ }^{*} g(x) \approx 0\right\}$ for some $x \in$ psns $^{*} X$. Thus $M=\{f \in C(v X): f[x]=0\}$.

\section{THE DiAgONAL FUnCTION}

Let $H \subset C(X, Y)$ be a completely regular family endowed with a topology $\tau$. R. McCoy started in $[18,19]$ a systematic study of the so-called diagonal function (or the evaluation identification)

$$
\Delta: X \rightarrow F(H, Y), \quad \Delta(x)(f):=f(x) \quad \text { with } x \in X, f \in H .
$$

We emphasize the analogy of (8) to the embedding of a locally convex vector space into the bidual. If $\tau$ is finer than the pointwise topology then $\Delta(x)$ is actually in $C(H, Y)$. If $\sigma$ is a topology on $F(H, Y)$ or $C(H, Y)$ then it is very natural to investigate continuity properties of $\Delta$. Assume that $\sigma$ lies between $\tau_{p}$ and $\tau_{k}$. As in the proof of Theorem 3.2 it is easy to see that $\Delta$ is continuous if

(9) $f \in \operatorname{cpt}^{*} H, f_{0} \in H$ with $f \approx_{\tau} f_{0}$ and $x \approx x_{0}$ implies $f(x) \approx f_{0}\left(x_{0}\right)$.

If $\tau$ is a weakly conjoining topology on $H \subset C(X, Y)$ then $\Delta$ is continuous. Our next theorem generalizes results in [19].

7.1. Theorem. Let $Y$ be a Hausdorff space and $H \subset C(X, Y)$ be a completely regular family endowed with a weakly conjoining topology $\tau \supset \tau_{p}$. Then $\Delta$ : $X \rightarrow C(H, Y)$ is an embedding for every topology $\sigma$ on $C(H, Y)$ between $\tau_{p}$ and $\tau_{k}$. For each $\phi \in H$ we can extend $\phi \circ \Delta^{-1}: \Delta(X) \rightarrow Y$ to a continuous function $\tilde{\phi}: C(H, Y) \rightarrow Y$.

Proof. Since $X$ is Hausdorff and $H$ is a completely regular family $H$ separates the points of $X$, hence $\Delta$ is injective. Now let ${ }^{*} \Delta(x) \approx_{\sigma} \Delta\left(x_{0}\right)$ for $x \in{ }^{*} X$, $x_{0} \in X$. Since $\tau_{p} \subset \sigma$ we infer ${ }^{*} f(x)={ }^{*} \Delta(x)\left({ }^{*} f\right) \approx \Delta\left(x_{0}\right)(f)=f\left(x_{0}\right)$ for every $f \in H$. Assume that $x$ is not in $m\left(x_{0}\right)$. Then there exists an open neighborhood $U$ of $x_{0}$ with $x \notin{ }^{*} U$. Choose $f \in H$ with $f(X \backslash U)=\left\{y_{0}\right\}$ and $f\left(x_{0}\right)=y_{1}$. Then ${ }^{*} f(x)={ }^{*} y_{0}$, but $f\left(x_{0}\right)=y_{1}$, a contradiction. Thus $\Delta$ is an embedding. Now let $\phi \in H$. We want to show that $\phi \circ \Delta^{-1}: \Delta(X) \rightarrow Y$ can be extended continuously to a function $\tilde{\phi}: C(H, Y) \rightarrow Y$. For $L: H \rightarrow Y$ we define $\tilde{\phi}(L):=L(\phi)$. Then $\tilde{\phi}$ is an extension since $\tilde{\phi}(\Delta(x))=\Delta(x)(\phi)=$ $\phi(x)=\phi \circ \Delta^{-1}(\Delta(x))$. For the continuity of $\tilde{\phi}$ let $L \in{ }^{*} C(H, Y), L_{0} \in$ 
$C(H, Y)$ with $L \approx_{\sigma} L_{0}$. Since $\tau_{p} \subset \sigma$ we have $L\left({ }^{*} \phi\right) \approx L_{0}(\phi)$ for every $\phi \in H$. Hence ${ }^{*} \tilde{\phi}(L) \approx \tilde{\phi}\left(L_{0}\right)$. The proof is complete.

Now we want to describe the closure of $\Delta(X)$ in $F(H, Y)$ with respect to the topology $\sigma$. It is clear that $F \in \overline{\Delta(X)}^{\sigma}$ iff there exists $x \in{ }^{*} X$ with ${ }^{*} \Delta(x) \approx_{\sigma}$ $F$. For the topology $\tau_{p}$ of pointwise convergence this is equivalent to ${ }^{*} f(x) \approx$ $F(f)$ for all $f \in H$. Thus $x$ is necessarily in $H$-ns ${ }^{*} X$. Conversely, for ${ }^{*} \Delta(x):{ }^{*} H \rightarrow{ }^{*} Y$ with $x \in H$-ns ${ }^{*} X$ we can define the standard part function st $^{*} \Delta(x): H \rightarrow Y($ cf. $\S 2)$ and obviously ${ }^{*} \Delta(x) \approx_{p}$ st $^{*} \Delta(x)$. Thus $\overline{\Delta(X)}^{p}=$ $\left\{\right.$ st $^{*} \Delta(x): x \in H$-ns $\left.{ }^{*} X\right\}$. Another interesting question is whether the closure $\overline{\Delta(X)}^{p}$ in $F(H, Y)$ is automatically in $C(H, Y)$. Our result strengthens a result in [19].

7.2. Theorem. Let $\tau$ be the closed-open topology on $H \subset C(X, Y)$ and $Y$ regular. Then $\Delta(X) \subset F(H, Y)$ is evenly continuous. In particular, the closure of $\Delta(X)$ with respect to $\tau_{p}$ in $F(H, Y)$ is a subset of $C(H, Y)$.

Proof. $\Delta(X)$ is evenly continuous iff each ${ }^{*} \Delta(x)$ with $x \in{ }^{*} X$ satisfies the following condition: for every $y \in Y, f \approx_{\tau} f_{0} \in H$ with ${ }^{*} \Delta(x)\left(f_{0}\right) \approx y$ implies ${ }^{*} \Delta(x)(f) \approx y$. Equivalently:

$$
f \approx{ }_{\tau} f_{0} \text { and }{ }^{*} f_{0}(x) \approx y \text { imply } f(x) \approx y .
$$

Let us prove formula (10) for the closed-open topology: Let $U$ be an open neighborhood of $y$. Choose $V$ open with $y \in V \subset \bar{V} \subset U$. Then $f_{0}^{-1}(\bar{V})=: A$ is closed and $[A, U]$ is a neighborhood of $f_{0}$. Thus $f \in{ }^{*}[A, U]$, i.e., that $f\left({ }^{*} A\right) \subset{ }^{*} U$. Since $f_{0}(x) \approx y$ we have $x \in{ }^{*} A$. Thus $f(x) \in{ }^{*} U$ for every neighborhood $U$ of $y$.

Let now $Y=\mathbf{R}$ and $X$ be a completely regular Hausdorff space. Endow $H=$ $C(X)$ or $H=C^{b}(X)$ with the closed-open topology and $F(H, \mathbf{R})$ with the topology of pointwise convergence. Then $\Delta: X \rightarrow C(H, \mathbf{R})$ is an embedding and the pointwise closure $\overline{\Delta(X)}^{p}$ in $F(H, \mathbf{R})$ is contained in $C(H, \mathbf{R})$. As pointed out in [19] it follows from well-known results that $\overline{\Delta(X)}^{p}$ is $v(X)$ for $H=C(X)$ and $\beta(X)$ for $H=C^{b}(X)$. A more direct nonstandard proof runs as follows: we know that st ${ }^{*} \Delta: H$-ns ${ }^{*} X \rightarrow \overline{\Delta(X)}^{p} \subset \mathbf{R}^{H}$ is surjective. Then it is not very difficult to see that st $^{*} \Delta: H$-ns ${ }^{*} X / \approx_{\beta} \rightarrow \overline{\Delta(X)}^{p}$ is a (well-defined) homeomorphism.

\section{REFERENCES}

1. S. Albeverio, J. E. Fenstad, R. Høegh-Krohn, and T. Lindstrøm, Nonstandard methods in stochastic analysis and mathematical physics, Academic Press, Orlando, Fla., 1986.

2. R. Arens and J. Dugundji, Topologies for function spaces, Pacific J. Math. 1 (1951), 5-31.

3. J. P. R. Christensen, Topology and Borel structure, North-Holland, Amsterdam, 1974.

4. H. H. Corson and E. Michael, Metrizability of certain countable unions, Illinois J. Math. 8 (1964), 351-360.

5. J. C. Dyre, Nonstandard characterizations of ideals in $C(X)$, Math. Scand. 50 (1982), 44-54.

6. P. Ezust, Join continuity of function spaces, Colloq. Math. 21 (1970), 87-89.

7. J. M. G. Fell, A Hausdorff topology for the closed subsets of a locally compact non-Hausdorff space, Proc. Amer. Math. Soc. 13 (1962), 472-476. 
8. J. Flachsmeyer, Verschiedene Topologisierungen in Raume der abgeschlossenen Mengen, Math. Nachr. 26 (1963/64), 321-337.

9. G. Fox and P. Morales, A non-Hausdorff Ascoli Theorem for $k_{3}$-spaces, Proc. Amer. Math. Soc. 39 (1973), 633-636.

10. L. Gillman and M. Jerison, Rings of continuous functions, Springer-Verlag, New York, 1976.

11. R. E. Hoffmann, On weak Hausdorff spaces, Arch. Math. 32 (1979), 487-504.

12. A. E. Hurd and P. A. Loeb, An introduction to nonstandard real analysis, Academic Press, Orlando, Fla., 1985.

13. J. L. Kelley, General topology, Van Nostrand, Princeton, N. J., 1955.

14. H. J. Keisler, An infinitesimal approach to stochastic analysis, Mem. Amer. Math. Soc. No. 297 (1984).

15. P. Lambrinos, A topological notion of boundedness, Manuscripta Math. 10 (1973), 289-296.

16. __ Exponential laws and compact-open topology, Continuous Lattices and Their Applications (Bremen, 1982), Lecture Notes in Pure and Appl. Math., Vol. 101, Dekker, New York, 1985, pp. 181-191.

17. M. Machover and J. Hirschfeld, Lectures on non-standard analysis, Lecture Notes in Math., Vol. 94, Springer, Berlin, 1969.

18. R. A. McCoy, The evaluation identification and the Ascoli theorem, Indian J. Math. 28 (1986), 195-201.

19. _ The evaluation identification in function spaces, Topology Appl. 11 (1980), 187-197.

20. R. A. McCoy and I. Ntantu, Topological properties of spaces of continuous functions, Lecture Notes in Math., vol. 1315, Springer-Verlag, Berlin, 1988.

21. E. Michael, $\aleph_{0}$-spaces, J. Math. Mech. 15 (1966), 983-1002.

22. __ Compact-covering images of metric spaces, Proc. Amer. Math. Soc. 37 (1973), 260266.

23. M. G. Murdeshwar, General topology, Wiley Eastern Limited, New Dehli, 1983.

24. L. Narens, Topologies of closed subsets, Trans. Amer. Math. Soc. 143 (1969), 393-411.

25. N. Noble, Ascoli theorems and the exponential map, Trans. Amer. Math. Soc. 143 (1969), 393-411.

26. H. Render, Topologische Untersuchungen von Funktionenräumen mit Nichtstandardmethoden, Dissertation, Duisburg, 1989.

27. __ Local compactness of function spaces, Arch. Math. 56 (1991), 286-287.

28. __ Generalized uniform spaces and applications to function spaces (submitted).

29. C. E. Rickart, General theory of Banach algebras, Van Nostrand, New York, 1960.

30. R. E. Smithson, First countable hyperspaces, Proc. Amer. Math. Soc. 56 (1976), 325-328.

31. L. A. Steen and J. A. Seebach, Counterexamples in topology, 2nd ed., Springer-Verlag, New York, 1978.

32. K. D. Stroyan and W. A. J. Luxemburg, Introduction to the theory of infinitesimals, Academic Press, New York, 1976.

33. F. Wattenberg, Topologies on the set of closed subsets, Pacific J. Math 68 (1977), 537-551.

34. D. E. Wulbert, Subsets of first countable spaces, Proc. Amer. Math. Soc. 19 (1968), 12731277.

Fachbereich Mathematik der Universität Duisburg, Lotharstr. 65, D-4100 Duisburg 1, Germany

E-mail address: render@math.uni-duisburg.de 